\title{
Defective Vortex Lattices in Layered Superconductors with Both Point and Correlated Pins
}

\author{
J. P. Rodriguez ${ }^{1}$ \\ ${ }^{1}$ Dept. of Physics and Astronomy, California State University, \\ Los Angeles, California 90032, USA.
}

(Dated: November 20, 2018)

\begin{abstract}
The mixed phase of layered superconductors with no magnetic screening is studied through a partial duality analysis of the corresponding $X Y$ model in the presence of random pinning centers. Sufficently weak Josephson coupling between adjacent layers results in an entangled vortex solid that exhibits weak superconductivity across layers. The corresponding vortex liquid state shows an inverted specific heat anomaly that is a precursor to the weak superconductor.
\end{abstract}

keywords : vortex-lattice pinning, dimensional cross-over, fishtail. 


\section{INTRODUCTION}

The possibility that the vortex lattice of a pristine layered superconductor with external magnetic field oriented perpendicular to the layers decouples into an incoherent stack of two-dimensional (2D) vortex lattices before it melts has been proposed many times in the literature 1] [2] [3]. Monte Carlo simulations and a duality analysis of the corresponding layered $X Y$ model with uniform frustration that describes the extreme type-II limit find that the decoupled vortex lattice state does not exist in practice [4] [5], however. The presence of random pinning centers [6] [7] result in quenched-in entanglements of the vortex lines that can reverse this conclusion [8].

In this paper, we shall show that random point pins drive a dimensional cross-over transition in the zero-temperature limit between defective vortex lattice states that exhibit weak versus strong superconductivity across layers. The magnetic field at the dimensional crossover transition notably decreases with an increase in the level of random point pinning. This is consistent with recent experimental determinations of how the "fishtail" feature that is shown by the irreversible magnetization of organic superconductors in magnetic field oriented perpendicular to the layers moves with a change in the level of material disorder [9]. We also find that the introduction of correlated pins can act to increase the dimensional cross-over field in the zero-temperature limit. A similar effect has been recently shown by the dimensional cross-over field extracted from flux creep dynamics in high-temperature superconductors 10]. Last, we find that the zero-temperature cross-over in magnetic field is inextricably linked to the existence of an inverted specific heat anomaly within the vortex liquid phase 8]. The latter accounts for the experimental observation of a similar thermal anomaly inside the vortex liquid phase of the high-temperature superconductor YBCO 11].

\section{THEORY}

We shall describe the mixed phase of layered superconductors with random pins by the corresponding $X Y$ model with uniform frustration over the cubic lattice. Both fluctuations of the magnetic induction and of the magnitude of the superconducting order parameter are neglected within this approximation. The model is therefore valid deep inside the interior of the mixed phase. The thermodynamics of the three-dimensional (3D) $X Y$ model with 
anisotropy and with uniform frustration is determined by the superfluid kinetic energy

$$
E_{X Y}^{(3)}=-\left.\sum_{\mu=x, y, z} \sum_{r} J_{\mu} \cos \left[\Delta_{\mu} \phi-A_{\mu}\right]\right|_{r},
$$

which is a functional of the superconducting phase $\phi(r)$ over the cubic lattice. The local phase rigidities within layers, $J_{x}$ and $J_{y}$, are assumed to be constant over most of the nearest-neighbor links, with the exception of those links in the vicinity of a pinning site, while the Josephson coupling $J_{z}$ on links between adjacent layers is assumed to be constant and weak. The vector potential $A_{\mu}=\left(0,2 \pi f x / a,-b_{\|} x\right)$ represents the magnetic induction, with a component $B_{\perp}=\Phi_{0} f / a^{2}$ oriented perpendicular to the layers, and with a component $B_{\|}=\left(\Phi_{0} / 2 \pi d\right) b_{\|}$oriented parallel to the layers. Here $a$ denotes the square lattice constant for each layer, which is of order the zero-temperature coherence length, while $d$ represents the separation between layers. Also, $\Phi_{0}$ denotes the flux quantum, and $f$ denotes the concentration of planar vortices per site.

Partial Duality Analysis. The layered $X Y$ model (11) can be effectively analyzed in the selective high-temperature limit, $k_{B} T \gg J_{z}$, through a partial duality transformation [3] . This leads to a dilute Coulomb gas ( $\mathrm{CG}$ ) ensemble that describes the nature of the Josephson coupling in terms of dual charges that live on the vertical links. In particular, thermodynamic quantities are obtained by taking appropriate derivatives of the logarithm of the partition function

$$
Z_{X Y}^{(3)}[0]=\left[I_{0}\left(J_{z} / k_{B} T\right)\right]^{\mathcal{N}^{\prime}} \cdot Z_{\mathrm{CG}}[0] \cdot \Pi_{l} Z_{X Y}^{(2)}[0]
$$

for the layered superconductor, expressed here as a product of the partition function for a layered CG ensemble [3]

$$
Z_{\mathrm{CG}}[0]=\sum_{\left\{n_{z}(r)\right\}} y_{0}^{N\left[n_{z}\right]} \cdot \Pi_{l} C_{l}\left[q_{l}\right] \cdot e^{-i \sum_{r} n_{z} A_{z}},
$$

with the contributions from each layer $l$ in isolation, $Z_{X Y}^{(2)}$, along with a modified Bessel function, $I_{0}(x)$, raised to the total number of links between adjacent layers, $\mathcal{N}^{\prime}$. Above, the dual charge, $n_{z}(\vec{r}, l)$, is an integer field that lives on links between adjacent layers $l$ and $l+1$ located at 2D points $\vec{r}$. The CG ensemble is weighted by a product of phase auto-correlation functions

$$
C_{l}\left[q_{l}\right]=\left\langle\exp \left[i \sum_{\vec{r}} q_{l}(\vec{r}) \phi(\vec{r}, l)\right]\right\rangle_{J_{z}=0}
$$


for isolated layers $l$ probed at the dual charge that accumulates onto that layer:

$$
q_{l}(\vec{r})=n_{z}(\vec{r}, l-1)-n_{z}(\vec{r}, l)
$$

It is also weighted by a bare fugacity $y_{0}$ that is raised to the power $N\left[n_{z}\right]$ equal to the total number of dual charges, $n_{z}= \pm 1$. The fugacity is given by $y_{0}=J_{z} / 2 k_{B} T$ in the selective high-temperature regime, $J_{z} \ll k_{B} T$, reached at large model anisotropy.

Defective Vortex Lattices. A recent analysis of the 2D limit of the $X Y$ model (10), $J_{z}=0$, finds that a phase coherent vortex lattice state with quenched-in unbound dislocations [12] exists in the zero-temperature limit if the random pinning is weak enough 8$]$. This defective vortex lattice state exhibits $2 \mathrm{D}$ phase auto-correlation functions (4) of the form $C_{l}[q]=$ $\left|C_{l}[q]\right| \exp \left[i \sum_{\vec{r}} q(\vec{r}) \phi_{0}(\vec{r}, l)\right]$, where the magnitude decays algebraicly like

$$
\left|C_{l}[q]\right|=g_{0}^{n_{+}} \cdot \exp \left[\eta_{2 D} \sum_{(1,2)} q(1) \ln \left(r_{12} / r_{0}\right) q(2)\right]
$$

asymptotically, and where $\phi_{0}(\vec{r}, l)$ represents the zero-temperature configuration of layer $l$ in isolation. Above, $\eta_{2 D}$ is the correlation exponent that vanishes linearly with temperature in the zero-temperature limit, and $r_{0}$ is the natural ultraviolet scale. Also, $g_{0}$ is equal to the ratio of the $2 \mathrm{D}$ phase rigidity with its value at zero temperature, while $n_{+}$is equal to half the number of probes in $q(\vec{r})$. At this stage it becomes useful to re-express the layered CG enesemble (3) by replacing $C_{l}[q]$ with its magnitude (6), and by compensating this change with the additional replacement of $-A_{z}(\vec{r}, l)$ with

$$
\phi_{l, l+1}^{(0)}(\vec{r})=\phi_{0}(\vec{r}, l+1)-\phi_{0}(\vec{r}, l)-A_{z}(\vec{r}, l) .
$$

A Hubbard-Stratonovich transformation of the CG partition function (3) then reveals 13] that it is equal to the partition function for a renormalized Lawrence-Doniach (LD) model, $Z_{\mathrm{LD}}[0]=\int \mathcal{D} \theta e^{-E_{\mathrm{LD}} / k_{B} T}$, up to a factor that is independent of the Josephson coupling, $J_{z}$. The LD energy functional is given by [3]

$$
E_{\mathrm{LD}}=\rho_{s}^{(2 D)} \int d^{2} r \sum_{l}\left[\frac{1}{2}\left(\vec{\nabla} \theta_{l}\right)^{2}-\Lambda_{0}^{-2} \cos \theta_{l, l+1}\right],
$$

where $\theta_{l, l+1}=\phi_{l, l+1}^{(0)}+\theta_{l+1}-\theta_{l}$. The $2 \mathrm{D}$ phase rigididity above is related to the $2 \mathrm{D}$ correlation exponent by $\rho_{s}^{(2 D)}=k_{B} T / 2 \pi \eta_{2 D}$. Also, $\Lambda_{0}=\gamma^{\prime} a$ is the Josephson penetration depth, where $\gamma^{\prime}=\left(J / J_{z}\right)^{1 / 2}$ is the model anisotropy parameter with respect to the macroscopic phase 
rigidity of an isolated layer at zero-temperature, $J$. A standard thermodynamic analysis of the product of partition functions (22) then yields that the strength of the local Josephson coupling is given by $\overline{\left\langle\cos \phi_{l, l+1}\right\rangle}=y_{0}+g_{0} \overline{\left\langle\cos \theta_{l, l+1}\right\rangle}$. Here

$$
\phi_{l, l+1}(\vec{r})=\phi(\vec{r}, l+1)-\phi(\vec{r}, l)-A_{z}(\vec{r}, l)
$$

is the gauge-invariant phase difference across adjacent layers, while the overbar notation represents a bulk average. It can also be shown [3] that the phase rigidity across a macroscopic number of layers is equal to $\rho_{s}^{\perp} / J_{z} \cong g_{0} \overline{\left\langle\cos \theta_{l, l+1}\right\rangle}$.

To compute the bulk average $\overline{\left\langle\cos \theta_{l, l+1}\right\rangle}$ at low temperature, we must first determine the configuration $\theta_{l}^{(0)}(\vec{r})$ that optimizes $E_{\mathrm{LD}}$. Eq. (8) implies that it satisfies the field equation

$$
-\nabla^{2} \theta_{l}^{(0)}+\Lambda_{0}^{-2}\left[\sin \theta_{l-1, l}^{(0)}-\sin \theta_{l, l+1}^{(0)}\right]=0 .
$$

In the weak-coupling limit, $\Lambda_{0} \rightarrow \infty$, we therefore have that $\theta_{l}^{(0)}(\vec{r})$ is constant inside of each layer. Also, by analogy with 2D melting physics 14] 15], the presence of quenched-in unbound dislocations in the pinned vortex lattice [8] [12] implies that the quenched-in interlayer order parameter $\exp i \phi_{l, l+1}^{(0)}$ shows only short-range correlations on average over the bulk. Specifically, we have

$$
\overline{\exp \left[i \phi_{l, l+1}^{(0)}(1)\right] \cdot \exp \left[-i \phi_{l, l+1}^{(0)}(2)\right]}=e^{i b_{\|} x_{12}} e^{-r_{12} / l_{\phi}}
$$

asymptotically, where $l_{\phi}$ is a $2 \mathrm{D}$ disorder scale set by the density of unbound dislocations between adjacent layers (see next section). The absence of long-range order along adjacent layers on average over the bulk then implies that $\overline{\cos \theta_{l, l+1}^{(0)}} \cong \overline{\left(\delta \theta_{l}^{(0)}-\delta \theta_{l+1}^{(0)}\right) \cdot \sin \theta_{l, l+1}^{(0)}}$ at zero temperature in the weak-coupling limit $[\underline{6}]$, where $\delta \theta_{l}^{(0)}$ is the deviation with respect to the constant background. This yields the perturbative result

$$
\overline{\cos \theta_{l, l+1}^{(0)}} \cong \Lambda_{0}^{-2} \int d^{2} r_{12} \overline{\exp \left[i \phi_{l, l+1}^{(0)}(1)\right] \cdot \exp \left[-i \phi_{l, l+1}^{(0)}(2)\right]} \cdot G^{(2)}\left(r_{12}\right)
$$

at weak coupling by Eq. (10), where $G^{(2)}=-\nabla^{-2}$ is the 2D Greens function. After taking the separation at which $G^{(2)}$ vanishes to be of order $\Lambda_{0}$, we then obtain the final perturbative result [7]

$$
\overline{\cos \theta_{l, l+1}^{(0)}} \cong\left[A_{0}-A_{2}\left(b_{\|} l_{\phi}\right)^{2}\right]\left(l_{\phi} / \Lambda_{0}\right)^{2} \ln \left(\Lambda_{0} / l_{\phi}\right)
$$

for the bulk average at zero temperature in the weak-coupling limit, and at weak parallel field $B_{\|} \ll \Phi_{0} / 2 \pi l_{\phi} d$. Here $A_{0}$ and $A_{2}$ are numerical constants of order unity: e.g., $A_{0}=1$ 
and $A_{2}=3 / 2$ if Eq. (111) is generally valid. By the discussion following Eq. (9), we conclude that random point pins result in a vortex glass that exhibits weak superconductivity across layers [3]], $\rho_{s}^{\perp} \ll J_{z}$, at sufficiently high layer anisotropy[8], $\Lambda_{0} \gg l_{\phi}$. Notice, however, that the quadratic dependence with small parallel field shown by the interlayer LD cosine (13) implies the absence of line tension for Josephson vortices! We believe that this reflects the vortex-glass nature of the phase coherence [2]: $\overline{\left\langle e^{i \phi_{l, l+1}(0)} e^{-i \phi_{l, l+1}(\infty)}\right\rangle}=0$ and $\overline{\left|\left\langle e^{i \phi_{l, l+1}(0)} e^{-i \phi_{l, l+1}(\infty)}\right\rangle\right|^{2}}>0$. Last, the bulk average at zero temperature $\overline{\cos \theta_{l, l+1}^{(0)}}$ must pass between zero and unity at $\Lambda_{0} \sim l_{\phi}$ as a function of the anisotropy parameter. This condition then defines a decoupling cross-over field

$$
B_{D}^{\perp}(0) \sim\left(l_{\phi} / a_{\mathrm{vx}}\right)^{2}\left(\Phi_{0} / \Lambda_{0}^{2}\right)
$$

at which point the reversible magnetization shows a broad diamagnetic peak. Above, $a_{\mathrm{vx}}=$ $a / f^{1 / 2}$ is the planar inter-vortex scale.

Decoupled Vortex Liquid. Suppose now that the macroscopic phase coherence (6) shown by each layer in isolation at low temperature is lost at temperatures above a transition temperature $T_{g}^{(2 D)}$, and that only short-range phase correlations over a scale $\xi_{2 D}$ exist. A high-temperature expansion 16] of the CG ensemble (3) in powers of the fugacity $y_{0}$ is then possible at temperatures above the $2 \mathrm{D}$ glass transition in the weak-coupling limit. At zero parallel field in particular, Eqs. (21) and (3) yield the expansion

$$
\begin{aligned}
& \overline{\left\langle\cos \phi_{l, l+1}\right\rangle} \cong y_{0} \sum_{1} \overline{C_{l}(0,1) \cdot C_{l+1}^{*}(0,1)}- \\
& y_{0}^{3}\left[\sum_{1,2,3} \overline{C_{l}(0,1) \cdot C_{l+1}^{*}(0,1) \cdot C_{l}(2,3) \cdot C_{l+1}^{*}(2,3)}-\sum_{1,2,3}{ }^{\prime} \overline{C_{l}(0,1,2,3) \cdot C_{l+1}^{*}(0,1,2,3)}\right]
\end{aligned}
$$

for the inter-layer "cosine" to next-leading order in the fugacity. The prime over the sum in the last term above excludes double counting of probes $(0,1,2,3)$. Also, next-leadingorder contributions that couple together three adjacent layers are negligible in the critical regime, $\xi_{2 D} \gg l_{\phi}$, and these are not listed above. Substitution of the form $C_{l}(1,2)=$ $g_{0} e^{-r_{12} / \xi_{2 D}} e^{i \phi_{0}(1)} e^{-i \phi_{0}(2)}$ for the phase autocorrelation function (44) into the leading term above yields a non-divergent result

$$
\overline{\left\langle\cos \phi_{l, l+1}\right\rangle} \sim g_{0}^{2}\left(J / k_{B} T\right)\left[\left(l_{\phi}^{-1}+\xi_{\phi}^{-1}\right)^{-1} / \Lambda_{0}\right]^{2}
$$


where $\xi_{\phi}=\xi_{2 D} / 2$. This implies an anomalous inter-layer contribution to the specific heat per volume equal to

$$
\delta c_{v}^{\perp} \cong 2\left[1+\left(\xi_{\phi} / l_{\phi}\right)\right]^{-1}\left(\partial \ln \xi_{\phi}^{-1} / \partial T\right) e_{J},
$$

where $e_{J}=\overline{\left\langle\cos \phi_{l, l+1}\right\rangle} \cdot J / \Lambda_{0}^{2} d$ is the Josephson energy density, and where $d$ denotes the spacing in between adjacent layers. It notably shows an inverted specific heat jump that is followed by a tailoff at a temperature $T_{p}$ such that $\xi_{\phi}\left(T_{p}\right) \sim l_{\phi}$ if $\xi_{\phi}$ diverges faster than $\left[T-T_{g}^{(2 D)}\right]^{-1}$.

The next-leading-order term for the inter-layer "cosine" (15) is negative, divergent, and of order $-y_{0}^{3} g_{0}^{4} l_{\phi}^{4} \xi_{\phi}^{2} / a^{6}$ in the critical region, $\xi_{\phi} \gg l_{\phi}$. This means that it becomes comparable to the leading order term (16) at magnetic fields below a cross-over scale

$$
B_{\times}^{\perp} \sim g_{0}\left(J / k_{B} T\right)\left(l_{\phi} \xi_{\phi} / a_{\mathrm{vx}}^{2}\right)\left(\Phi_{0} / \Lambda_{0}^{2}\right)
$$

that separates 2D from 3D vortex-liquid behavior [5] [8]. The phase correlation length across layers grows larger than the separation between adjacent layers at temperatures below this point, $T_{\times}$. It then diverges (or jumps to infinity) at a $3 \mathrm{D}$ ordering temperature, $T_{g}$, that lies inside of the window $\left[T_{g}^{(2 D)}, T_{\times}\right]$. The phase diagram displayed by Fig. 1 summarizes all of the above.

\section{DISCUSSION AND CONCLUSIONS}

The present theory predicts a disorder-driven dimensional crossover (14) between defective vortex lattices that show weak versus strong superconductivity across layers. Comparison of the bulk average (11) of the quenched-in phase autocorrelation with the corresponding thermal average in the hexatic phase of a pristine vortex liquid [14] indicates that the $2 \mathrm{D}$ disorder scale, $l_{\phi}$, that controls the crossover transition is set by the density of unbound dislocations between adjacent layers in the vortex lattice. For example, the addition or the removal of a straight line of dislocations that traverses all of the layers will not change this scale. A useful parameterization of $l_{\phi}$ is then

$$
l_{\phi}=l_{2 D} /\left(2 x_{\text {point }}^{1 / 2}\right),
$$

where $l_{2 D}$ is the correlation length of the quenched-in order parameter $\exp \left(i \phi_{0}\right)$ on average over a given layer in isolation, and where $x_{\text {point }}$ is the concentration of unbound dislocations 
between adjacent layers relative to the total number of unbound dislocations in adjacent layers: e.g., $x_{\text {point }}=0$ if all of the unbound dislocations form smooth lines across layers, while $x_{\text {point }}=1$ if sections of such lines are absent. Eqs. (14) and (19) then yield two important predictions: the decoupling crossover field $B_{D}^{\perp}(0)$ is $(i)$ diminished by an increase in the density of unbound dislocations between adjacent layers, $l_{\phi}^{-2}$, and it (ii) increases with an increase in the concentration of unbound dislocations that line-up across adjacent layers, $1-x_{\text {point }}$. Prediction $(i)$ is consistent with a recent proposal that the second magnetization peak (or "fishtail") observed in the mixed phase of organic superconductors is due to dimensional crossover of the vortex lattice [9]. The second-peak shown by the irreversible magnetization that is measured in this work moves to lower perpendicular magnetic field as the concentration of random point pins increases. Also, prediction (ii) is consistent with recent experiments that observe a 2D-3D crossover in the flux creep dynamics of hightemperature superconductors in magnetic field oriented perpendicular to the layers [10]. The dimensional cross-over field that is extracted experimentally in ref. [10] is found to increase after columnar pins are introduced. It is quite possible that these help to line-up a fraction of the existing unbound dislocations across layers, which in turn leads to an increase in the decoupling field (14) as a result of the increase in the $2 \mathrm{D}$ disorder scale $l_{\phi}$.

The second important result obtained here is the inverted specific-heat anomaly, Eq. (17), that is predicted to exist inside of the decoupled vortex liquid phase. Recent experiments on 2D arrays of Josephson junctions with bond disorder in external magnetic field are consistent with a continuous vortex glass transition that has a $2 \mathrm{D}$ phase correlation length that diverges as $\xi_{2 D} \propto\left[T-T_{g}^{(2 D)}\right]^{-\nu_{2 D}}$ at the transition, with a critical exponent $\nu_{2 D}=2$ [17]. This implies an inverted specific heat anomaly, Eq. (17), that peaks at $\xi_{2 D}\left(T_{p}\right)=2 l_{\phi}$, which is shown in Fig. 2. A similar anomaly was observed within the vortex-liquid phase of YBCO 11]. Comparison of the magnitude of that anomaly with Eq. (177) yields a 10\% change in the interlayer cosine (16) across the vortex-liquid/vortex-liquid transition [8]. The above indicates that the specific-heat anomaly observed experimentally inside of the vortex-liquid phase of YBCO represents not a true phase transition [18], but a crossover instead.

Noticably absent from the above calculations is the effect of magnetic coupling between planar vortices across layers. This in general reduces the entanglement of vortex lines across layers, and it will therefore increase the inter-layer phase coherence, Eqs. (13) and (16). Ref. [7] shows, however, that the effect of magnetic coupling can be accounted for by 
a renormalization up in the $2 \mathrm{D}$ disorder scale, $l_{\phi}$. Last, a sharp and possibly first-order disorder-driven decoupling transition has been found numerically at zero temperature for the vortex lattice with magnetic interactions, but with no Josephson coupling [19]. This indicates that the disorder-driven dimensional crossover found here in the zero-temperature limit survives the addition of weak magnetic coupling.

We conclude that random point pinning of the vortex lattice in layered superconductors results in a 2D-3D crossover transition at low temperature that separates strong from weak superconductivity across layers (see Fig. 1). The disorder-driven phenomenon suggests the following physical picture: although the tension for a line of dislocations in the defective vortex lattice is null on both sides of the crossover, the scale of the roughness in the line is large compared to the inter-layer spacing in the 3D phase, while it is of order or less than the inter-layer spacing in the quasi-2D phase. Scaling suggests that such quenched-in disorder is established first at separations between layers of order $l_{\phi} / \gamma$, where $\gamma=\Lambda_{0} / d$ is the physical anisotropy parameter.

The author thanks C. Reichhardt and C. Olson for informative discussions.

[1] L.I. Glazman and A.E. Koshelev, Phys. Rev. B 43, 2835 (1991); M. Feigel'man, V.B. Geshkenbein, and A.I. Larkin, Physica C 167, 177 (1990); E. Frey, D.R. Nelson, and D.S. Fisher, Phys. Rev. B 49, 9723 (1994).

[2] D.S. Fisher, M.P.A. Fisher and D.A. Huse, Phys. Rev. B 43, 130 (1991).

[3] J.P. Rodriguez, Phys. Rev. B 62, 9117 (2000).

[4] A.E. Koshelev, Phys. Rev. B 56, 11201 (1997).

[5] J.P. Rodriguez, Phys. Rev. B 66, 214506 (2002); Erratum: Phys. Rev. B 69, 069901 (2004).

[6] A.E. Koshelev, L.I. Glazman and A.I. Larkin, Phys. Rev. B 53, 2786 (1996).

[7] L.N. Bulaevskii, M.B. Gaifullin, Y. Matsuda and M.P. Maley, Phys. Rev. B 63, 140503 (2001).

[8] J.P. Rodriguez, Phys. Rev. B 69, 100503(R) (2004).

[9] N. Yoneyama, T. Sasaki, T. Nishizaki and N. Kobayashi, J. Phys. Soc. Jpn. 73, 184 (2004).

[10] D.G. Marinaro, J. Horvat, S.X. Dou, R. Weinstein and A. Gandini, Phys. Rev. B 68, 064518 (2003).

[11] F. Bouquet, C. Marcenat, E. Steep, R. Calemczuk, W.K. Kwok, U. Welp, G.W. Crabtree, 


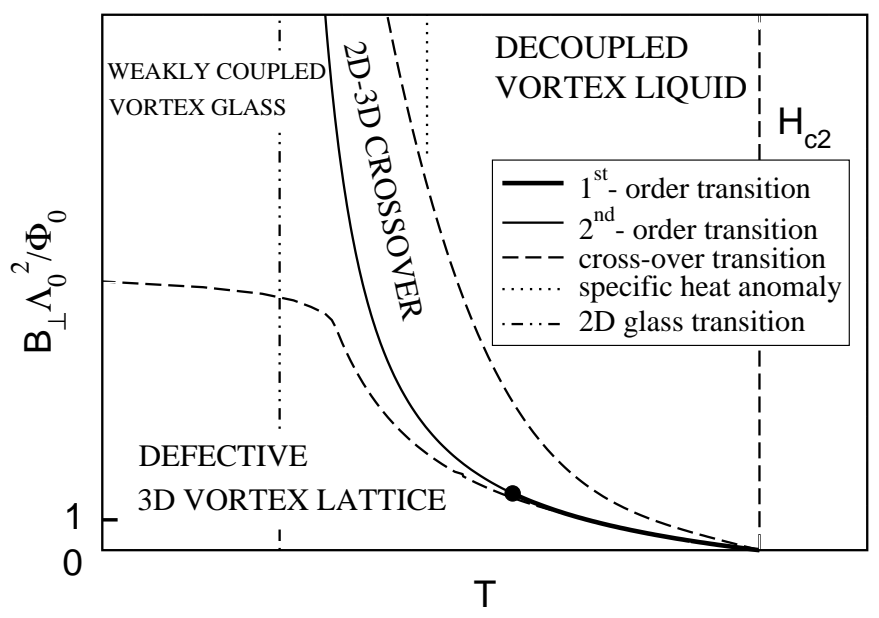

FIG. 1: Shown is a proposed phase diagram assuming weak point pins and a continuous vortex glass phase transition for isolated layers. The perpendicular magnetic field is held fixed while the Josephson penetration depth, $\Lambda_{0}$, is swept along the verticle axis. A mean-field temperature dependence, $J \propto T_{c 0}-T$, is also assumed.

R.A. Fisher, N.E. Phillips and A. Schilling, Nature 411, 448 (2001).

[12] E.M. Chudnovsky, Phys. Rev. B 43, 7831 (1991).

[13] J.P. Rodriguez, J. Phys. Cond. Matter 9, 5117 (1997).

[14] J.P. Rodriguez, Phys. Rev. Lett. 87, 207001 (2001).

[15] D.R. Nelson and B.I. Halperin, Phys. Rev. B 19, 2457 (1979).

[16] A.E. Koshelev, Phys. Rev. Lett. 77, 3901 (1996).

[17] Y.-J. Yun, I.-C. Baek and M.-Y. Choi, Phys. Rev. Lett. 89, 037004 (2002).

[18] Z. Tesanovic, Phys. Rev. B 59, 6449 (1999).

[19] C.J. Olson, G.T. Zimanyi, A.B. Kolton and N. Gronbech-Jensen, Phys. Rev. Lett. 85, 5416 (2000). 


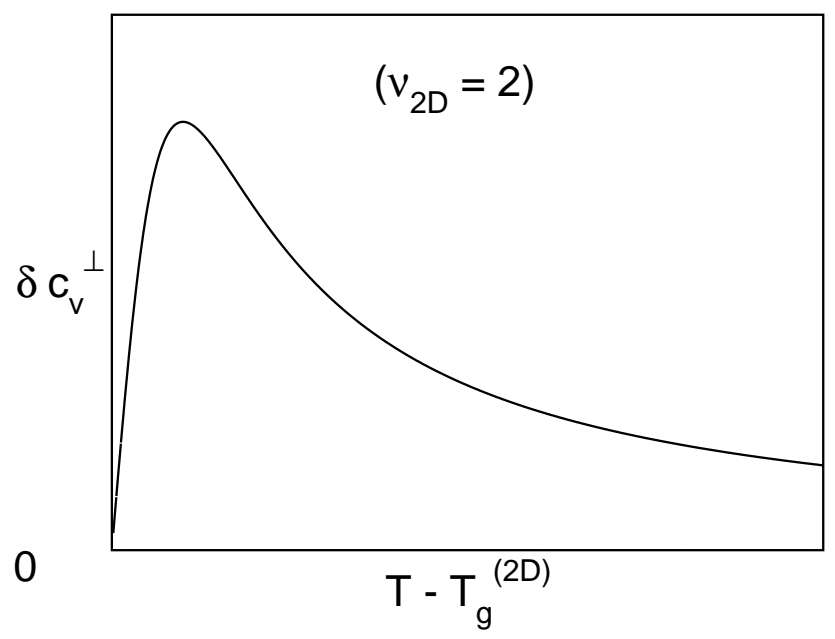

FIG. 2: Theoretical specific heat anomaly, Eq. (17), for $\xi_{2 D} \propto\left[T-T_{g}^{(2 D)}\right]^{-2}$. The peak occurs at $\xi_{2 D}\left(T_{p}\right)=2 l_{\phi}$ 